\title{
Anticancer Therapy and Dentition: An Overlooked Late Adverse Effect in Childhood Cancer Survivors
}

\author{
Srinivasan Peyam ${ }^{1} \cdot$ Deepak Bansal $^{1}[$
}

Received: 19 December 2021 / Accepted: 21 December 2021/Published online: 18 January 2022

c) Dr. K C Chaudhuri Foundation 2021

With improving outcomes of childhood cancers, increasing interest is being directed towards the long-term adverse effects in childhood cancer survivors (CCSs). Dental developmental disturbances (DDDs) are receiving attention given the adverse effect on the quality of life and optimal nutrition. Anticancer therapy (ACT) constituting systemic chemotherapy and radiotherapy to the head and neck region can interfere with odontogenesis resulting in several aberrations, including tooth agenesis, tooth discoloration, root shortening, or malformation, reduced salivary flow, dental caries, enamel hypoplasia, and microdontia. The DDDs can be irreversible $[1,2]$. Children are typically affected, while the adults receiving ACT are spared as the dental structures are already formed.

The risk factors for DDDs include (a) age below 3 y while receiving ACT, (b) dose-dependent alkylating agents including cyclophosphamide $\left(>4 \mathrm{~g} / \mathrm{m}^{2}\right)$, and (c) radiotherapy dose exceeding $20 \mathrm{~Gy}$ to head and neck region [3, 4]. Few recent studies have suggested age below $4 \mathrm{y}$ and radiotherapy dose exceeding 20 Gy to predict DDDs better than the dose of radiotherapy alone $[4,5]$. In the current protocols for acute lymphoblastic leukemia (ALL), prophylactic cranial radiotherapy is no longer included. In this issue of the Journal, Atif et al. report the long-term adverse effect of ACT on dentition in CCSs from a single center in New Delhi [6]. They have demonstrated the adverse effects of the early age of initiation of ACT ( $<5 \mathrm{y})$ on DDDs [6]. It is in concordance with earlier studies $[1,3,4]$. In addition, the authors demonstrate that microdontia is a common crown defect observed in $41 \%$ of CCSs, similar to the findings of earlier studies $[3,7,8]$.

Deepak Bansal

deepakbansaldr@gmail.com

1 Pediatric Hematology-Oncology Unit, Department of Pediatrics, Advanced Pediatrics Center, Postgraduate Institute of Medical Education and Research, Chandigarh 160012, India
In a recent survey study by a Dutch group, root defects were more common than crown defects, with impaired root growth and microdontia being the most common root and crown defects, respectively, in CCSs [4]. The root abnormalities, including shortening, blunting, tapering, and the etiology of clinically missing teeth, including impacted tooth and tooth agenesis, can be better studied by orthopantomogram (OPG) [7, 9]. In a systematic review and meta-analysis of 16 nonrandomized studies, root defects were observed in $62 \%$ of cases using OPG [7]. The commonest abnormality noted was impaired root growth in $58 \%$ of survivors, and the commonest cancer was leukemia in $45 \%$ of cases [7].

In the study by Atif et al., nearly half the study population (45\%) is constituted by ALL survivors [6]. It was an opportunity for analyzing the various root defects, the effect of alkylating agents, and the cumulative doses on dental anomalies in CCSs. The dose, site, and age of administration of radiotherapy are not analyzed. Also, understandably, the OPG was performed in a few selected cases. The effect of cumulative dose of alkylating agents and the dose, site, and age at administration of radiotherapy on DDDs has not been specifically addressed. There is a need to model a risk profile to identify the CCSs at the greater risk of DDDs. It would help to target the at-risk survivors for dental referral, early identification, and appropriate intervention for dental adverse effects. Likely, the sample size and heterogeneous patient population were the limiting factors in the study by Atif et al. to identify comprehensive risk factors. Notably, there is a void in the literature on the adverse effect of intensity-modulated radiotherapy on dental effects [1].

Chemotherapy and radiotherapy can also lead to xerostomia, which, combined with enamel hypoplasia, results in an increased risk of dental caries. Probably, xerostomia was not evaluated as the indications for radiotherapy to the head and neck region are limited in childhood cancers compared to adults. Dental caries is a common finding in clinical practice. Both these parameters could have been addressed in the study of Atel et al., as it is helpful when planning 
orthodontics. Xerostomia is observed to be associated with periodontal disease. It is postulated to be associated with cardiovascular disease, which can be compounded by anthracyclines and radiotherapy $[1,10]$.

In low- and middle-income countries (LMICs), universal availability of cancer survivor clinics and ready access to dentists with expertise in CCSs is a distant reality. Indeed, there is an unmet need to identify at-risk survivors for early intervention. LMICs account for $80 \%-90 \%$ of childhood cancer burden, providing an opportunity for designing a scoring system to predict the risk of DDDs in CCSs. The study by Atif et al. is one of the very few studies that has addressed dental health in CCSs, particularly in LMICs.

\section{Declarations}

\section{Conflict of Interest None.}

\section{References}

1. Gawade PL, Hudson MM, Kaste SC, et al. A systematic review of dental late effects in survivors of childhood cancer. Pediatr Blood Cancer. 2014;61:407-16.

2. Busenhart DM, Erb J, Rigakos G, Eliades T, Papageorgiou SN. Adverse effects of chemotherapy on the teeth and surrounding tissues of children with cancer: A systematic review with metaanalysis. Oral Oncol. 2018;83:64-72.

3. Kaste SC, Goodman P, Leisenring W, et al. Impact of radiation and chemotherapy on risk of dental abnormalities: a report from the Childhood Cancer Survivor Study. Cancer. 2009;115:5817-27.

4. Stolze J, Vlaanderen KCE, Holtbach FCED, et al. Long-term effects of childhood cancer treatment on dentition and oral health: a dentist survey study from the DCCSS LATER 2 study. Cancers (Basel). 2021;13:5264.

5. Milgrom SA, van Luijk P, Pino R, et al. Salivary and dental complications in childhood cancer survivors treated with radiation therapy to the head and neck: a pediatric normal tissue effects in the clinic (PENTEC) comprehensive review. Int J Radiat Oncol Biol Phys. 2021. https://doi.org/10.1016/j.ijrobp.2021.04.023.

6. Atif M, Mathur VP, Tewari N, Bansal K, Rahul M, Bakhshi S. Long-term effect of anticancer therapy on dentition in childhood cancer survivors: an observational, cross-sectional study. Indian J Pediatr. 2021. https://doi.org/10.1007/s12098-021-03818-1.

7. Seremidi K, Kloukos D, Polychronopoulou A, Kattamis A, Kavvadia K. Late effects of chemo and radiation treatment on dental structures of childhood cancer survivors. A systematic review and meta-analysis. Head Neck. 2019;41:3422-33.

8. Immonen E, Nikkilä A, Peltomäki T, Aine L, Lohi O. Late adverse effects of childhood acute lymphoblastic leukemia treatment on developing dentition. Pediatr Blood Cancer. 2021;68:e29200.

9. Ritwik P. Dental care for patients with childhood cancers. Ochsner J. 2018;18:351-7.

10. Teles R, Wang CY. Mechanisms involved in the association between periodontal diseases and cardiovascular disease. Oral Dis. 2011;17:450-61.

Publisher's Note Springer Nature remains neutral with regard to jurisdictional claims in published maps and institutional affiliations. 